\title{
Histopathological Fate in the Inguinal Hernia Sac in the Children
}

\section{Mustafa Yaşar Özdamar}

\author{
Faculty of Medicine, Department of Pediatric Surgery, Erzincan Bin Ali Yıldırım University, Erzincan, Turkey
}

*Corresponding author: Mustafa Yaşar Özdamar, Faculty of Medicine, Department of Pediatric Surgery, Erzincan Binali Yıldırım University, Başbağlar Mah 24100 Erzincan, Turkey, Tel: +90-446-226-18-18, Fax: +90-446-226-18-19

\begin{abstract}
Background/Purpose: Obliteration of the processus vaginalis during the fetal growth begins with a transient decrease in sympathetic tonus and then ends with smooth muscle cells (SMCs) undergoing apoptosis. Otherwise, an inguinal hernia $(\mathrm{IH})$ occurs due to the defective obliteration. Although the mechanism in the formation of an inguinal hernia has been elucidated by many investigations, it has not been investigated whether proliferation in the $\mathrm{IH}$ sac cells which would lead to the benign or malignant process. In this study, we aimed to examine whether the proliferative changes in the cells of $\mathrm{IH}$ sac would be a histopathological precursor to any process.
\end{abstract}

Methods: We obtained fifty (male 25, female 25) samples of the IH sac from patients (study group) during operations. Control groups were formed from twelve parietal peritoneum samples (male 4, female 8) undergoing laparotomy for various reasons, and two processus vaginalis samples obtaining from 2 boys. Samples were evaluated regarding mesothelial, smooth muscle, neuronal, adipose, and vascular tissue proliferation as well as inflammation and congestion. Histopathological evaluation was made with H\&E staining. Immunohistochemical evaluation was performed with PCNA and $\mathrm{HBME}-1$ known the proliferative and the mesothelial marker, respectively.

Results: There were significantly increased mesothelial, vascular, nerve, and SMC proliferation in the study groups compared with those of control groups $(p<0.05)$. The expression of HBME-1 and PCNA was significantly increased $(p<0.05)$ in control groups compared with those of controls.

Conclusions: We determined that the proliferation of SMCs increased in the $\mathrm{IH}$ sac as in previous studies. We also found the increased proliferation in the mesothelial cells together with the increased HBME-1 and PCNA expressions in the IH sac. These alterations may be an initiator of a benign or malignant process by which would be able to develop in the $\mathrm{IH}$ sac.

\section{Keywords}

Inguinal hernia, Sac, Proliferation, PCNA, HBME-1, Children

\section{Introduction}

Inguinal hernias in the children that require a semi-urgent operation due to the risk of incarceration can rarely lead to complications such as testis ischemia. From complications, the development of mesothelioma in a sac is an unusual process regarding the proliferation, which can be thought of as an endpoint during the developmental process of an IH sac [1-4].

Processus vaginalis (PV) forming with a peritoneal protrusion in the eighth week of pregnancy is wholly obliterated after the testis and ovary complete their descent to normal localization. If PV obliteration does not occur, it is inevitable that an inguinal hernia, hydrocele, and cord cysts or Nuck's duct cyst form. PV obliteration occurs through apoptosis, namely programmed cell death. Smooth muscle cells (SMCs), which is a specific marker in the structure of fetal PV, are not found in both peritoneal area and PV in the postnatal period. Tanyel, et al. suggested that the obliteration of the PV takes place through a transient decrease in sympathetic tonus and an increase in parasympathetic tonus in this process [4-8].

During obliteration of PV, the intracellular CAMP increase that causes a trophic effect on SCMs via $\beta$-adrenergic receptor due to increased sympathetic tonus ceases critical apoptosis by preventing dedifferentiation of SCMs. Also, increased sympathetic tonus is associated with increased androgen effect, and increased androgens act trophic impact on SCMs. As the PV affected by deviations relating the programmed cell death in these complicated steps, this situation shows that PV is not just a simple peritoneal protuberance $[9,10]$.

Tanyel, et al. asserted that myofibroblasts inducing 
apoptosis of SMCs in the fusion of mesothelial opposing layers in the PV lead to contribute to the obliteration [11]. In previous studies, immunohistochemical methods have been used to explain the indirect inguinal hernia mechanisms that occur in developmental aberrations in the PV [7-11]. The theories based on the absence of obliteration in the PV have focused on defective apoptosis through the persistence of fetal SMCs in the proliferative changes occurring in a hernia sac. Although the mechanism in the formation of an inguinal hernia has been elucidated by many investigations, it has not been investigated whether proliferation in the IH sac cells which would lead to the benign or malignant process. In this study, we aimed to examine whether the proliferative changes in the cells of $\mathrm{IH}$ sac would be a histopathological precursor to any process.

\section{Materials and Methods}

\section{Study subjects}

We obtained fifty (male 25, female 25 ) samples of the hernia sacs (study groups) from the one-sided indirect inguinal hernia operations between January 2016 and January 2018. Standard procedure was high ligation of the $\mathrm{IH}$ sac and ligation via monofilament absorbable sutures during operation. Control groups were formed from twelve parietal peritoneum samples which were obtained from patients (male 4, female 8 ) undergoing laparotomy for various reasons (two processus vaginalis samples obtained from 2 boys undergoing contra-lateral exploration were included in the male control group). The histological features of each group of samples were examined by a pathologist blinded to the specific pathology. The study was approved by the Institutional Ethics Review Board for Clinical Research (2018/24/4).

\section{Histopathological evaluation}

Immediately following the surgical procedure, specimens were placed in a neutral buffered formaldehyde solution for histopathological evaluation. Following the fixation of the examples, paraffin sections were stained with Masson trichrome (HT15; Sigma, Milan, Italy) and hematoxylin-eosin (H\&E) for microscopic evaluation. H\&E-stained sections of the control groups and the study groups were evaluated regarding mesothelial, smooth muscle, neuronal, adipose, and vascular tissue proliferation. All specimens were also examined concerning inflammation and congestion.

\section{Immunohistochemical evaluation}

For the comparative evaluation of the specimens through immunohistochemical, the glass slides were washed thoroughly with distilled water for 20 minutes and exposed to antigen for a total period of 20 minutes, with a one-minute break at five-minute intervals, in a target retrieval solution, which was diluted by $1 / 10$. The solution was left for twenty minutes at room temperature and then washed with distilled water. It was then kept for five minutes in phosphate-buffered saline (PBS). Then, it was incubated for one hour with $1 / 50$ diluted ready-for-use PCNA antibody (Code: SC-56, Santa Cruz Biotechnology, Inc.).

The rabbit anti-human HBME-1 (Hector Battifora mesothelial antigen-1) polyclonal antibodies were used as a marker of the mesothelial cells (diluted 1:50; cat. no. MA5-12220; Thermo Fisher Scientific, USA). The procedure was carried out in strict accordance with the instructions of rabbit anti-human HBME-1 monoclonal antibody kit. After it was incubated for about $15 \mathrm{~min}$ utes in a biotin solution, it was kept for 10 minutes in PBS. Then, it was incubated for about 15 minutes in a streptavidin-peroxidase solution. It was kept for five minutes in AEC (3 amino-9-etylcarbazole) chromogen after it was kept for 10 minutes in PBS. It was washed with distilled water. Cross-sections were then kept for five minutes in Mayer's hematoxylin for opposite staining. Afterward, it was flushed with tap water and enclosed with a mounting medium (Entellan, Merck Millipore, Darmstadt, Germany).

For immunostaining, a fully automated IHC device (Leica Bond-Max, Melbourne, Australia) was used. Histopathological and immunohistochemical evaluations were carried out using a light microscope (Olympus BX53, Tokyo, Japan).

\section{Staining score}

In the histopathological evaluation; the severity of mesothelial, smooth muscle, vascular and adipose tissue proliferation as well as inflammation and congestion were scored: 0 score $=$ no proliferation, 1 score $=$ mild $(+), 2$ scores $=$ moderate $(++)$ and, 3 scores $=$ strong $(+++)$ proliferation. Immunohistochemical evaluation showed nuclear staining especially in specimens stained with PCNA antibody. PCNA staining score was used for evaluating the proliferating activity of the mesothelial and other cells. Positive staining with HBME-1 was detected by yellow-brown granular staining, mostly in the cytoplasm and less in the cell membrane. No staining with nuclear membrane HBME-1 was observed. In the microscopic evaluation, 4 high-power fields were determined for each slice at the 4 corners and the center to calculate the proportion of positive cells of 100 sample cells; mean positive score was obtained from the 4 calculation. The percentage of positive cells was categorized on a scale of score $0=$ no staining, score $1=$ weak staining (less than $10 \%$ focal involvement), score $2=$ moderate staining (11-50\% regional involvement), and score 3 = strong staining (greater than $50 \%$ diffuse involvement) [12].

\section{Statistical analysis}

In all statistical analysis of the study, SPSS (version 16.0; SPSS Inc, Chicago, USA) was used. All parameters were stated as mean \pm S.D. Scores with ordinal data were compared between the groups by the Mann-Whit- 

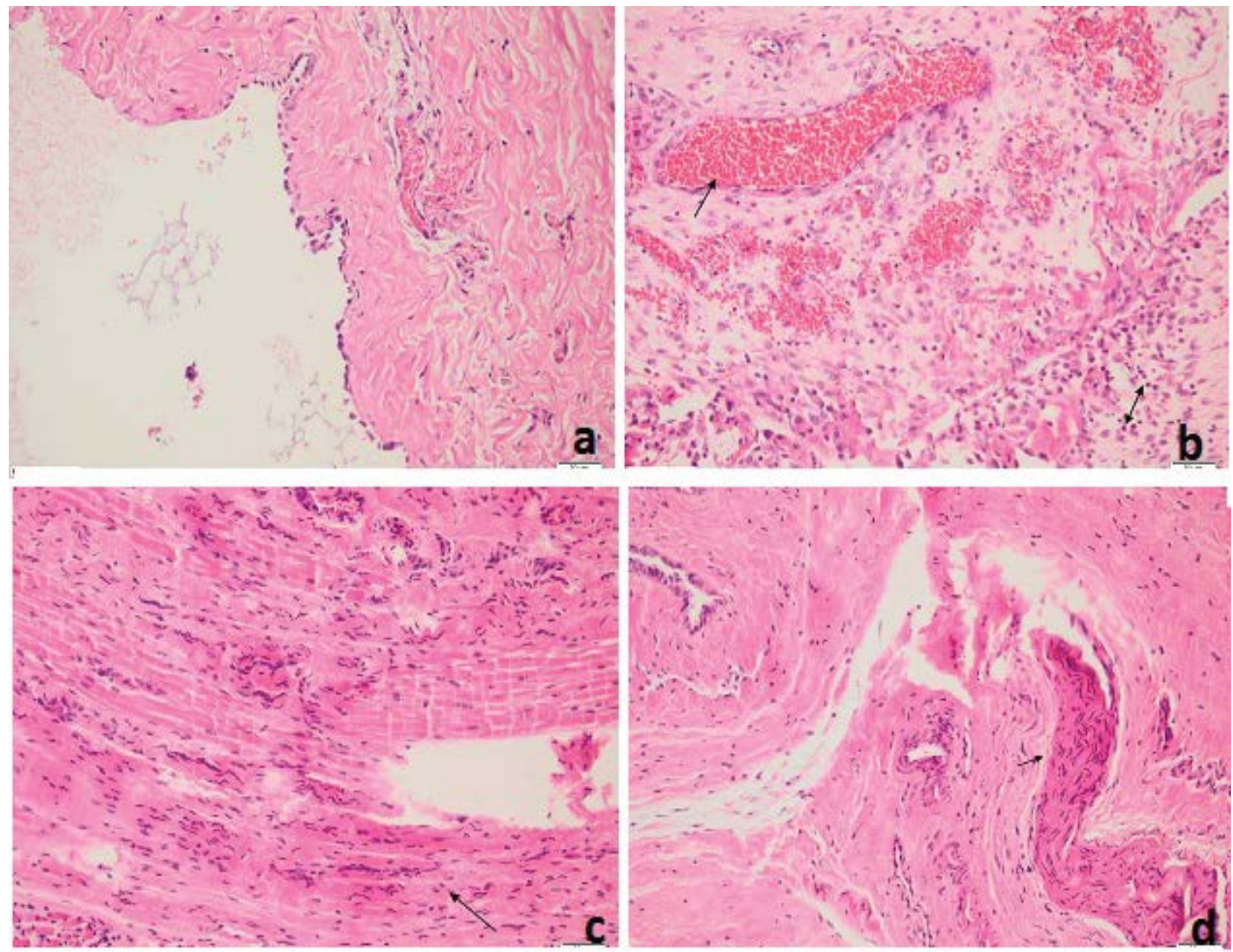

Figure 1: Histological findings (H\&E, orginal magnifation X 200); a) Normal peritoneum consisting of a superficial mesothelial layer and a submesothelial zone; b) Inguinal sac tissue with increased mesothelial proliferation, vascularity, inflammation and congestion; c) Increased smooth muscle proliferation; d) Marked neuronal tissue.

ney $U$ test. From the non-parametric tests, Mann-Whitney $U$ was used for statistical analysis because groups consisted of less than 30 cases and the data did not have a normal distribution in the groups according to the Shapiro-Wilk test. A possibility level of 0.05 or less was accepted to represent statistical significance.

\section{Results}

There was no significant difference between groups in terms of age: the group of male inguinal hernias $(\mathrm{MIH}), 43$ \pm 16 ; the group of female inguinal hernias $(\mathrm{FIH}), 43 \pm 24$; the group of male control (MC), $58 \pm 10$; and the group of female control $(F C), 56 \pm 13$ months $(p=0.00)$.

Histopathological examination of the sacs in the MIH and FIH groups revealed that the innermost layer had mesothelial cells, while there were blood vessels and peripheral nerves in the soft connective tissue outside this layer. There were also SMCs outside of this area. Histopathological examination of the peritoneum and $\mathrm{PV}$ in the control groups showed the same findings, but no SMCs were found (Figure 1). There were significantly increased mesothelial, vascular, nerve, adipose and SMC proliferation in the MIH group compared to MC (Figure 1$)$, which were statistically significant $(p<0.05)$.
There was an increased proliferation score in FIH group compared to FC, which were also statistically significant $(p<0.05)$. There was no significant difference between $\mathrm{FIH}$ and $\mathrm{MIH}$ except for neuronal tissue proliferation histopathologically when both groups were compared with each other. Namely, the neuronal staining score in males was higher than that of females $(p=0.00)$. The histopathological staining scores of the study $(\mathrm{MIH}, \mathrm{FIH})$ and the control (MC, FC) groups were shown in Table 1.

The expression of HBME-1 and PCNA (Figure 2) was significantly increased $(p<0.05)$ in the $\mathrm{MIH}$ and $\mathrm{FIH}$ groups compared with those of controls. The comparative evaluation of staining score regarding inflammation and congestion between the study and control groups showed significant variation $(p<0.05)$ in favor of the study groups (Figure 1), whereas there were no significant differences between MIH and FIH groups $(p>0.05)$. Histopathological (H\&E; inflammation, and congestion) and immunohistochemical (HBME-1 and PCNA) staining scores of the study $(\mathrm{MIH}, \mathrm{FIH})$ and control $(\mathrm{MC}, \mathrm{FC})$ groups were shown in Table 2.

\section{Discussion}

The PV composes of a process of the peritoneum 
Table 1: The histopathological findings with scores in the study (inguinal hernia sac samples) and control (PV and peritoneal samples) groups.

\begin{tabular}{|c|c|c|c|c|c|c|c|}
\hline $\begin{array}{l}\text { Type of tissue } \\
\text { proliferation }\end{array}$ & $\begin{array}{l}\text { MC score, } \\
\text { Mean } \pm \text { SD } \\
(n=6)\end{array}$ & $\begin{array}{l}\text { FC score, } \\
\text { Mean } \pm \text { SD } \\
(n=8)\end{array}$ & $\begin{array}{l}\text { MIH score, } \\
\text { Mean } \pm \text { SD } \\
(n=25)\end{array}$ & $\begin{array}{l}\text { FIH score, } \\
\text { Mean } \pm \text { SD } \\
(n=25)\end{array}$ & $\begin{array}{l}p, \\
\text { MC vs. MIH }\end{array}$ & $\begin{array}{l}p, \\
\text { FC vs. FIH }\end{array}$ & $\begin{array}{l}p \\
\text { MIH vs. FIH }\end{array}$ \\
\hline Mesothelial & $1.16 \pm 0.40$ & $1.12 \pm 0.35$ & $1.80 \pm 0.57$ & $1.96 \pm 0.73$ & 0.016 & 0.005 & 0.069 \\
\hline Smooth muscle & $1.33 \pm 0.51$ & $1.37 \pm 0.51$ & $2.32 \pm 0.69$ & $2.16 \pm 0.68$ & 0.005 & 0.008 & 0.013 \\
\hline Neuronal & $0.66 \pm 0.81$ & $0.62 \pm 0.74$ & $2.44 \pm 0.66$ & $1.36 \pm 0.63$ & 0.001 & 0.015 & 0.000 \\
\hline Vascular & $1.16 \pm 0.40$ & $1.25 \pm 0.46$ & $2.36 \pm 0.70$ & $2.40 \pm 0.60$ & 0.002 & 0.000 & 0.088 \\
\hline Adipose & $0.66 \pm 0.10$ & $0.25 \pm 0.11$ & $0.80 \pm 0.20$ & $0.76 \pm 0.13$ & 0.026 & 0.010 & 0.735 \\
\hline
\end{tabular}

MC: male control group; FC: female control group; MIH: group of male inguinal hernia; FIH: group of female inguinal hernia.

Table 2: Inflammation, congestion and, expressed HBME-1 and PCNA scores of the groups.

\begin{tabular}{|c|c|c|c|c|c|c|c|}
\hline & $\begin{array}{l}\text { MC score, } \\
\text { Mean } \pm \text { SD } \\
(n=6)\end{array}$ & $\begin{array}{l}\text { FC score, } \\
\text { Mean } \pm \text { SD } \\
(n=8)\end{array}$ & $\begin{array}{l}\text { MIH score, } \\
\text { Mean } \pm \text { SD } \\
(n=25)\end{array}$ & $\begin{array}{l}\text { FIH score, } \\
\text { Mean } \pm \text { SD } \\
(n=25)\end{array}$ & $\begin{array}{l}p, \\
\text { MIH vs. MC }\end{array}$ & $\begin{array}{l}p, \\
\text { FIH vs. FC }\end{array}$ & $\begin{array}{l}p, \\
\text { MIH vs. FIH }\end{array}$ \\
\hline Inflammation & $0.16 \pm 0.01$ & $0.25 \pm 0.16$ & $1.08 \pm 0.90$ & $1.16 \pm 0.94$ & 0.022 & 0.010 & 0.799 \\
\hline Congestion & $0.50 \pm 0.14$ & $0.62 \pm 0.51$ & $1.78 \pm 0.89$ & $1.64 \pm 0.86$ & 0.001 & 0.004 & 0.734 \\
\hline HBME-1 & $1.00 \pm 0.00$ & $1.00 \pm 0.00$ & $1.96 \pm 0.61$ & $1.88 \pm 060$ & 0.001 & 0.000 & 0.640 \\
\hline PCNA & $1.00 \pm 0.00$ & $1.00 \pm 0.00$ & $1.88 \pm 0.66$ & $1.84 \pm 0.62$ & 0.003 & 0.001 & 0.843 \\
\hline
\end{tabular}

MC: male control group; FC: female control group; MIH: group of male inguinal hernia; FIH: group of female inguinal hernia.

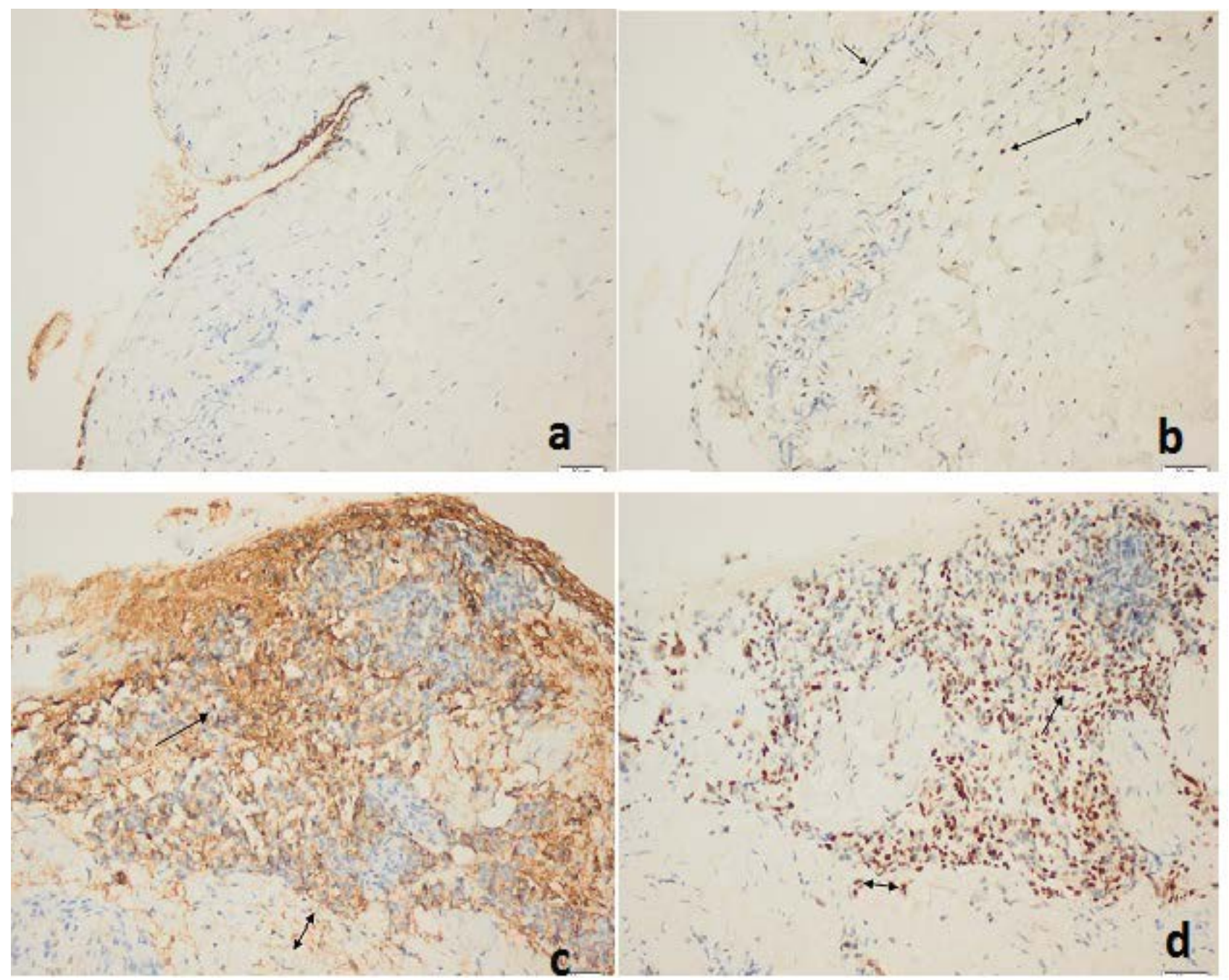

Figure 2: Immunohistochemical findings (original magnification X 200). In the control groups (a,b); a) Immunohistochemical slight staining of HBME-1 in mesothelial cells; b) Immunohistochemical slight staining of PCNA in mesothelial and stromal nuclear; c) Diffuse staining with HBME-1 of mesothelium and the submesothelial stromal area in the study group; d) Diffuse staining of mesothelium and stromal cells with PCNA in the study group. 
developing inside the gubernacular mesenchyme as the caudal genito-inguinal ligament. In the gubernacular mesenchyme; the outer side includes the cremaster muscle. In addition, while the invaginating PV takes form in the middle layer, the innermost is formed the cord that joins to the caudal epididymis and testis [13]. Amount of the persisting SMCs in the sac is vital for the clinical outcome. While the SMC is less present in hydrocele, the amount of SMC is the least in the sac associated with undescended testis. Additionally, there is more the smooth muscle bundle in the hernia sac $[5,9]$. Apoptosis of SMC is necessary for PV obliteration to take place. There, however, is scarce information in the literature about the fate of other PV cell structures other than SMC $[5,7,9,11]$. Tanyel, et al. suggested that the least SMCs were detected in the sac associated with undescended testis and hydrocele while the most SMCs were detected in the inguinal hernia sac. In parallel, they stated that the programmed cell death (PCD) was more observed in the sacs with the least SMC and PCD was higher in the cells such as mesothelial other than the SMC [14]. In our study, the proliferation of mesothelial, nerve, vascular and adipose tissues in the sacs increased in $\mathrm{MIH}$ and $\mathrm{FIH}$ groups compared to control groups ( $p<0.05$; Figure 1 and Table 1 ). There were significantly SMCs in the study groups while the SMCs were not encountered in the control groups. There was no significant difference between $\mathrm{MIH}$ and $\mathrm{FIH}$ groups regarding SMC proliferation ( $p>0.05)$.

As in previous studies, significant SMC proliferation in our histopathological evaluation with quantitative scores through H\&E staining was noticeable in the IH sacs. Unlike previous studies [5,9], there was also a marked increase in proliferation in cells other than SMCs. That is, PCD was probably defective in these cells or apoptosis did not occur after a certain period in these cells. In our study, increased expression of mesothelial HBME-1 which is the marker for mesothelial cells [15] was demonstrated to be a significant increase immunohistochemically in the $\mathrm{MIH}$ and $\mathrm{FIH}$ groups compared to the peritoneal and PV samples. HBME-1 was present mostly in the cytoplasm and less in the cell membrane. Also, the expression of PCNA significantly increased in the study groups compared to the control groups. PCNA is an essential protein found in proliferating cells, and it performs crucial roles in DNA replication, repair, and control of cell proliferation [16]. Immunohistochemical evaluation showed the increased nuclear staining with PCNA antibody in the study groups compared to those of control groups. This result together with the increased HBME-1 staining in the cytoplasm and cell membrane has seemed to demonstrate an increase in the proliferation of, especially mesothelial cells.

Patients who had no apparent history of asbestos exposure, but mesothelioma developing from the Nuck's canal, inguinal hernia, and hydrocele sacs have been reported in the literature $[3,4,17,18]$. As in PV, a compen- satory cell proliferation does not develop after developmental apoptosis or PCD [19]. If apoptosis does not occur in SMCs via PCD in the PV, an inguinal hernia, hydrocele, or abnormal testicular localization take place [5]. That is, there is no proliferation increase in mesothelial cells in obliterated PV as it was in our study. On the other hand, in the present work, we showed not only an increase in mesothelial cell proliferation via the increased in HBME1 and PCNA expression but also the increased in SMC proliferation qualitatively in the IH sac. On the contrary, some researchers have argued that both the $\mathrm{IH}$ sac and the obliterated PV share similar histopathology except for the amount of SMCs when examined qualitatively by optical microscopy using H\&E stain or indirect immunohistopathological method $[5,19]$. In a previous study, apoptotic nuclei have been detected within the vascular structures and mesothelium, but not within the SMCs in the hernia sac. Namely, the attempt of apoptosis during the process of PV obliteration has been shown in vascular and mesothelial cells. Based on the results we had, therefore, it may be speculated that when the obliteration of PV through SMCs apoptosis or PCD did not occur, the mesothelial cell together with vascular and neuronal cells underwent proliferation because of incomplete apoptosis in the same failure process. Even though inguinal hernia surgery in children is delayed due to some social reasons, any practitioner does not encounter a malignancy that develops from the hernia sac. In the literature, however, mesothelioma case arising from $\mathrm{IH}$ sac have been encountered in patients who were at adult ages [1-4,17,18]. Therefore, the increased mesothelial proliferation in the $\mathrm{IH}$ sac would seem to be a predecessor of such malignancy for IH patients at very low rates theoretically in any period of their life.

The mechanism by which occurs PV obliteration via SMC apoptosis is identical to be responsible for the inhibition of testicular descent. That is, the process of failed testis descent remains the physiologic pathway for the obliteration of PV. While undescended testis may indicate the decrease of SMC requiring for propelling the testis through the PV via a premature activation of this process, prolonged exposure of the cremaster muscle to the parasympathetic tonus may cause to ascent an undescended testis. The reason for sustained contractility of the cremaster muscle is the increase in the level of cytosolic calcium [14].

Apoptosis of SM in the PV occurs through mitochondrial $\mathrm{Ca}^{2+}$ overload and then depletion of $\mathrm{Ca}^{2+}$ stores with an increase in cytosolic $\mathrm{Ca}^{2+}$. This cascade is initiated by an increase in Bax and Fas proteins regulating at the level of the mitochondrial membrane. Thus, the SMCs and mesothelium cells are exposed to PCD, respectively; and obliteration of the PV takes place $[8,14]$. Apoptosis in a cell occurs through two interconnected pathways. The extrinsic pathway is started by ligation of death-inducing receptors. The intrinsic pathway is initiated by oxidative stress, DNA damage, hypoxia or growth factor depriva- 
tion. This pathway is controlled by the $\mathrm{Bcl}-2$ superfamily of proteins at the level of the mitochondrial membrane [20]. The depletion of $\mathrm{Ca}^{2+}$ from internal stores through inositol 1,4,5-trisphosphate receptors (IP3) via activation of phospholipase $\mathrm{C}$ by $\mathrm{G}$-protein-linked signal transduction takes part in this initial steps in PCD [21]. Insulin-like growth factor-I (IGF-I) carries out many of its antiapoptotic actions through regulation of mitochondrial membrane permeability via $\mathrm{Bcl}-2$ proteins, thus working against apoptosis. While IGF-I increases $\mathrm{Bcl}-2$ interacting mediator of cell death via the intrinsic apoptotic pathway, it blocks the translocation to the mitochondrion of the proapoptotic protein Bax via the same apoptotic pathway [22,23]. In a recent study, it was detected IGF Receptor-1 (IGFR-1) in the cremaster muscle (CM) associated with both the undescended and the descended testis. The density of IGFR-1 was higher in the CM, CM's nerves and vascular tissues of the descended testis group than that of the undescended testis group [24]. The gubernacular-CM complex is innervated by the genitofemoral nerve (GFN). Alteration and divisions of the genitofemoral nerve inhibit the descent of the testis $[25,26]$. The $\mathrm{Bcl}-2$ family as a crucial key of neuronal cell fate plays a role in the antiapoptotic action at the level of varying from purely cytosolic to the predominantly mitochondrial membrane in the neuronal cell. The decreased activities of $\mathrm{Bcl}-2$ cause the decreased sympathetic tonus and lose of the neuron. Defective innervation of a muscle also gives rise to an increase in Bax and loss of motor neuron. As the IGFR-1 promotes the decrease of Bax and Fas and the increase $\mathrm{Bcl}-2$ proteins; the receptor deficiency leads to the increase in Bax and Fas and the decrease Bcl-2 [27]. This result may be indicative of the decreased expression of IGFR-1 in CM associated with undescended testis [24]. On the other hand, for this apoptotic pathway which is effective in the apoptosis of PV, it may be proposed that the activity of IGFR-1 is required. If there is a defect or density difference at the IGFR-1 receptor, there may be alterations in the apoptosis of PV and is the need for further investigations.

Some studies have demonstrated that IGF1 is required for the development and functioning of the LCS in the developmental process of the testis. Based on this information it can be said that the Leydig cells in which insulin-like hormone 3 (INSL-3) requiring for the testis descent is produced need IGF1; otherwise, the production of INSL-3 becomes defective [28]. Therefore, the low density of IGFR-1 in the gubernacular-CM complex may probably be effective in the etiology of undescended testis [24]. According to mentioned above information, the PV obliteration requires a decrease in $\mathrm{Bcl}-2$ and an increase in Bax for the SMCs apoptosis. IGFR-1 has a significant effect that triggers this event, and this act is accomplished through its receptor. Therefore, if PV and $\mathrm{CM}$ with the same mesenchymal origin are thought to share the same fate, the contribution of IGFR-1 to obliteration in the PV is likely.
In our study, there was no significant difference between FIH and MIH except for neuronal tissue proliferation histopathologically when both groups were compared. The neuronal staining score in males was higher than that of females $(p=0.00)$. This difference may be due to the sympathetic tonus being sexually dimorphic which is in favor of the male [10].

In a previous study, Nishioka, et al. showed that mesenchymal cells expressing vimentin appeared before mesothelial regeneration on the fibrotic peritoneum. Also, they found that HBME-1-positive cells were originated from vimentin-positive cells [15]. In another study, it has been suggested that immature SMCs known myofibroblast express vimentin, as an intermediate filament in the apoptosis of SMC which plays a role in the obliteration of the PV. Vimentin has been recognized, among other intermediate filaments, as an indicator of undifferentiated SMCs $[9,11]$. That is, if cells expressing vimentin represent both mesothelial and SMC precursor cells, vimentin-positive cells in the inguinal hernia sac may represent both. This result may be indicative of increased HBME-1 expression with a marker of mesothelial proliferation in the hernia sac as in our study. Also, increased PCNA expression in a hernia sac supports increased mesothelial cell proliferation.

\section{Conclusions}

The most popular etiological reason in the indirect $\mathrm{IH}$ is the defect in the PV obliteration. Defective apoptosis through the persistence of fetal SMCs in the proliferative changes occurring in the hernia sac gives rise to the defective obliteration of the PV. In our study, we determined that the SMCs increased in the hernia sac. This detection was supporting previous investigations and parallel with that of theirs. As it has been found in some recent works, PV and CM with the same mesenchymal origin are thought to share the same fate, the contribution of IGFR-1 to obliteration in the PV is likely. As a result of this defective apoptotic pathway, we also found an increase in proliferation of the mesothelial cells with the increased HBME- 1 and PCNA expressions. There are mesothelioma cases developed in the hernia sac in advanced adult ages in the literature. Therefore, we suppose that the increase in mesothelial proliferation in the IH sac would seem to be a predecessor of such malignancy.

\section{Acknowledgements}

I would like to thank Dr. Ilyas Sayar for his help in the histopathological and immunohistochemical evaluation and examination of this study.

\section{Conflicts of Interest}

None.

Funding

None. 


\section{References}

1. Ozdamar MY, Karakus OZ (2017) Testicular Ischemia Caused by Incarcerated Inguinal Hernia in Infants: Incidence, Conservative treatment procedure, and Follow-up. Urol J 14: 4030-4033.

2. Çakmak AM, Ateş U, Ekberli G (2018) Inguinal hernias and hydrocele: Etiology, diagnosis and treatment-management of incarcerated hernia. Turkiye Klinikleri Pediatric Surgery Special Topics 8: 1-12.

3. Chakravartty S, Singh JC, Jayamanne H, Shah V, Williams GL, et al. (2012) Peritoneal mesothelioma masquerading as an inguinal hernia. Ann R Coll Surg Engl 94: e111-e112.

4. Manatakis DK, Stamos N, Agalianos C, Vamvakas P, Kordelas A, et al. (2013) Mesothelial cyst of the round ligament misdiagnosed as irreducible inguinal herni. Case Rep Surg 2013: 408078.

5. Tanyel FC, Dağdeviren A, Müftüoğlu $S$, Gürsoy $M H$, Yürüker S, et al. (1999) Inguinal hernia revisited through comparative evaluation of peritoneum, processus vaginalis, and sacs obtained from children with hernia, hydrocele, and undescended testis. J Pediatr Surg 34: 552-555.

6. Glick PL, Boulanger SC (2006) Inguinal hernias and hydroceles. In: Grosfeld JL, O'Neill JA Jr, Fonkalsrud EW, Coran AG, Pediatric surgery. (6th edn), Elsevier, Philadelphia, $1172-1192$.

7. Tanyel FC, Kale G (2001) Evaluation of mystery of the hernia sac through another perspective. Pediatr Dev Pathol 4: 512-513.

8. Tanyel FC, Ulusu NN, Tezcan EF, Büyükpamukcu N (2003) Total calcium content of sacs associated with inguinal hernia, hydrocele or undescended testis reflects differences dictated by programmed cell death. Urol Int 70: 211-215.

9. Mouravas VK, Koletsa T, Sfougaris DK, Philippopoulos A, Petropoulos AS, et al. (2010) Smooth muscle cell differentiation in the processus vaginalis of children with hernia or hydrocele. Hernia 14: 187-191.

10. Tanyel FC (2004) Obliteration of processus vaginalis: Aberrations in the regulatory mechanism result in an inguinal hernia, hydrocele or undescended testis. Turk J Pediatr 46: 18-27.

11. Tanyel FC, Müftüoglu S, Dagdeviren A, Kaymaz FF, Büyükpamukçu N (2001) Myofibroblasts defined by electron microscopy suggest the dedifferentiation of smooth muscle within the sac walls associated with congenital inguinal hernia. BJU Int 87: 251-255.

12. Yirmibesoglu E, Karahacioglu E, Kilic D, Lortlar N, Akbulut $G$, et al. (2012) The protective effects of Ginkgo biloba extract (EGb-761) on radiation-induced dermatitis: An experimental study. Clin Exp Dermatol 37: 387-394.

13. Barteczko KJ, Jacob MI (2000) The testicular descent in human. Origin, development and fate of the GT Hunteri, processus vaginalis peritonei, and gonadal ligaments. Adv Anat Embryol Cell Biol 156: 1-98.
14. Tanyel FC, Okur HD (2000) Autonomic nervous system appears to play a role in obliteration of processus vaginalis. Hernia 8: 149-154.

15. Nishioka Y, Miyazaki M, Abe K, Furusu A, Harada T, et al. (2008) Regeneration of peritoneal mesothelium in a rat model of peritoneal fibrosis. Ren Fail 30: 97-105.

16. Zheleva DI, Zhelev NZ, Fischer PM, Duff SV, Warbrick E, et al. (2000) A quantitative study of the in vitro binding of the C-terminal domain of p21 to PCNA: affinity, stoichiometry, and thermodynamics. Biochemistry 39: 7388-7397.

17. Aggarwal M, Lakhar B, Shetty D, Ullal S (2000) Malignant peritoneal mesothelioma in an inguinal hernial sac: an unusual presentation. Indian J Cancer 37: 91-94.

18. Testini M, Scattone A, Di Venere B, Lissidini G, Piccinni G, et al. (2005) Primary malignant peritoneal mesothelioma in an incarcerated groin hernia: Report of a case. Surg Today 35: $421-424$

19. Fan Y, Bergmann A (2008) Apoptosis-induced compensatory proliferation. The Cell is dead. Long live the Cell! Trends Cell Biol 8: 467-473.

20. Van Gurp M, Festjens N, van LG, Saelens X, Vandenabeele $P$ (2003) Mitochondrial intermembrane proteins in cell death. Biochem Biophys Res Commun 304: 487-497.

21. Fuller GM, Shields D (1998) The cell cycle and cell division. In: Fuller GM, Shields D, Molecular Basis of Medical Cell Biology. Appleton \& Lange, Stamford, 106-123.

22. Leinninger GM, Russell JW, van Golen CM, Berent A, Feldman EL (2004) Insulin-like growth factor-I regulates glucose-induced mitochondrial depolarization and apoptosis in human neuroblastoma. Cell Death Differ 11: 885-896.

23. Ness JK, Scaduto Jr RC, Wood TL (2004) IGF-I prevents glutamate-mediated bax translocation and cytochrome $\mathrm{C}$ release in $\mathrm{O}^{4+}$ oligo-dendrocyte progenitors. Glia 46: 183194.

24. Özdamar MY, Şahin S, Zengin K, Seçkin S, Gürdal M (2018) Detection of insulin-like growth factor receptor-1 in the human cremaster muscle and its role in the etiology of the undescended testis. Asian J Surg.

25. Tanyel FC, Erdem S, Buyukpamukcu N, Tan E (2000) Cremaster muscles obtained from boys with an undescended testis show significant neurological changes. BJU Int 85: 116-119.

26. Clarnette TD, Hutson JM (1996) The genitofemoral nerve may link testicular inguinoscrotal descent with congenital inguinal hernia. Aust NZ J Surg 66: 612-627.

27. Akhtar RS, Ness JM, Roth KA (2004) Bcl-2 family regulation of neuronal development and neurodegeneration. Biochim Biophys Acta 1644: 189-203.

28. Manna PR, Chandrala SP, King SR, Jo Y, Counis R, et al. (2006) Molecular mechanisms of insulin-like growth factor-I mediated regulation of the steroidogenic acute regulatory protein in mouse leydig cells. Mol Endocrinol 20: 362-378. 